\title{
Bemerkungen zur jurakischen Lautlehre
}

Nach dem Erscheinen von T. Lehtisalos lange ersehntem Werke "Juraksamojedisches Wörterbuch" (Helsinki 1956) sind wir besser als vorher imstande, Untersuchungen über die jurakische Lautlehre wie über andere Seiten der jurakischen Sprache zu treiben. Die Aufzeichnungen dieses hervorragenden Erforschers des Jurakischen sind ja eigentlich das Einzige, was in genauer phonetischer Transkription aus dieser Sprache zul unserer Verfügung steht. Früher war man hauptsächlich auf seine umfangreichen Textaufzeichnungen hingewiesen, aber es ist sehr zeitraubend, die für lautliche Untersuchungen nötigen Daten aus Texten in vielen verschiedenen Dialekten herauszuholen, und die Lage ist also jetzt viel günstiger.

Meine Abhandlung "The form of the object in the Uralic languages" (Uppsala 1955) ist u.a. von Aulis J. Joki (FUF Anz. 32, S. 1-55) und von Péter Hajdú (NyK 59, s. 247-251) besprochen worden. Ich bin für diese Besprechungen sehr dankbar, da sie besonders auf dem Gebiete des Juraksamojedischen zu interessanten Diskussionen Anlass geben können. Da diese Sprache für die vergleichende uralische Sprachwissenschaft ausserordentlich wert voll und ergiebig aber noch viel zu wenig untersucht ist, habe ich es für angebracht gefunden, mit den folgenden Zeilen einen Versuch zu machen, einige Punkte der jurakischen Lautlehre zu beleuchten. Es hat sich nämlich aus den obenerwähnten Besprechungen herausgestellt, diss in grewissen wichtigen Punkten auch der deskriptiven Lilutlehr. verschiedene Heinungen bestehen. Da inzwischen das grosss Wörterbuch von T. Lehtisalo erschienen ist, will ich die (relegenheit ergreifen, mit Hilfe des dort gegebenen Materials die Diskussion weiterzuführen und womöglich die betreffenden Fragen zu klären, wobei ich auch auf ein paar Stellen gegen meine verehrten Kritiker ein wenig polemisieren muss, haupt- 
sächlich gegen Joki, der meine obenerwähnte Abhandlung einer detaillierten und teilweise recht strengen Kritik unterworfen hat. Die Fragen, die im Folgenden behandelt werden, gehören hauptsächlich zum Gebiet der deskriptiven Lautlehre, und sprachgeschichtliche Fragen werden nur vorübergehend berührt. Die hier besprochenen Verhältnisse werden sich aber wahrscheinlich in gewissen Punkten auch für die Sprachgeschichte als wichtig erweisen, da sie den Bau des jurakischen 1.i.ltsystems betreffen.

Bcim Zitieren der Wörter aus Lehtisalos Wörterbuch (im Folrenden WB verkül'zt) habe ich seine Schreibung hauptsächlich unverändert beibehalten. Einige kleine Vereinfachungen, die mir in diesem Zusammenhang belanglos scheinen aber das Drucken mid Iesen der Graphente etwas erleichtern, habe ich mir jedoch gestattet. So habe ich die unter einigen Konsonantenzeichen (besonders oft unter den Buchstiben $s$ und $t$ ) stehenden kleinen Bogen wegrelassen (und schreibe also $s$ statt $s$ usw.), und ebenso die unter Konsonintenzeichen stehenden nach unten gerichteten Pfeile (also $\chi$ st: $x$ tt $\chi$ usw.; unter Vokalzeichen werden sie aber beibehalten). Statt $i^{\text {s schreibe }}$ ich einfach $t$, aber $t^{\prime} s$ wird beibehalten. Das Zeichen für einen am Wortende stehenden überkurzen stimmlosen $e$-Laut wird weggelassen, und das doppelte Zeichen für den Kehlkopfverschluss wird einfach geschrieben, also z.B. $j \bar{\imath}$ statt $j \ddot{\imath} " \not$. Ebenso wird das bisweilen vorkommende Zeichen der Silbenscheide fortgelassen, und auch der Halbstimmhaftigkeit angebende Haken nach den Buchstaben für stimmlose Medien.

Die Bezeichnungen der jurakischen Dialekte sind die von Lehtisalo im WB verwendeten. Um die Darstellung nicht zu weitläufig zu machen, habe ich die Beispiele hauptsächlich aus einer und derselben Mundart genommen (und die Verhältnisse in anderen Dialekten nur hier und da kurz behandelt) und dabei das im WB am reichlichsten vertretene Idiom gewählt, nämlich das dort mit dem Signum $\mathrm{O}$ oder $\mathrm{O}_{1}$ bezeichnete, also die Obdorsker Mundart nach den von Maxim Jadoptsew gelieferten Mitteilungen. Die Bedeutungen der Belege werden oft nur ganz kurz wiedergegeben. 
Die meisten jurakischen Konsonanten kommen in zwei verschiedenen Gestalten vor, von denen die eine unmouilliert und die andere mouilliert ist, z.B. $t$ und $l, n$ und $n, p$ und $\dot{p}$. Der velare Nasal $\eta$ hat als Phonem deskriptiv betrachtet keine mouillierte Entsprechung (in solchen Fällen wie jē $\bar{q} \underline{\text { g }}$ 'Schritt' $\sim$ Akk.Pl. jèn'ś $i$ ist $\eta$ als eine kombinatorische Variante von $n$ zu betrachten), aber historisch betrachtet hat er in gewissen Fällen die mouillierte Entsprechung $\dot{n}$ (siehe die ausführliche Untersuchung von Hajdú in seiner Arbeit "Die sekundären anlautenden Nasale ( $\left.\eta^{-}, n^{-}\right)$im Samojedischen", Acta Linguistica IV, Budapest 1954). Auch der velare Verschlusslaut $k$ und der Kehlkopfverschluss ' kommen nur unmouilliert vor, die Frikativa $\chi$ dagegen wechselt paradigmatisch mit ś (z.B. jä $\chi \dot{a}$ 'Fluss' Akk.Pl. jëšśs $)$, das sonst die mouillierte Entsprechung von $s$ ist. Der Frikativa $\beta$ entspricht inlautend unter den mouillierten Konsonanten $b$ (oder $\dot{B}$ ), ebenso wie $d$ die mouillierte Entsprechung von $\delta$ ist, aber anlautend gibt es synchronisch betrachtet im Tundrajurakischen keine mouillierte Entsprechung von $\beta$ (obgleich historisch betrachtet wie bekannt $j$ als solcher auftritt, z.B. $j \bar{\imath}$ ' 'Wasser' $\sim$ waldjurakisch $\beta^{\prime} i t$ ), wenn wir von vereinzelten jungen Lehnwörtern absehen, wie Sjo R'orsp 'Fischreuse' (< russ.). Der palatale Konsonant $j$ wiederum, den wir hier zu den mouillierten Konsonanten rechnen werden, hat deskriptiv betrachtet keine unmouillierte Entsprechung.

Die Vokale $a, o, u$ treten nach einem mouillierten Konsonanten in einer anderen Gestalt auf als nach einem unmouillierten Konsonanten, und ihre Aussprache wird auch in gewissem Umfang von der mouillierten oder unmouillierten Beschaffenheit des nachfolgenden Konsonanten beeinflusst, wobei ein diphthongbildendes $i$ dieselbe Wirkung wie ein mouillierter Konsonant ausübt. Für die Vokale der ersten Silbe sollen jetzt mit Hilfe des Materials in Lehtisalos WB einige Beobachtungen mitgeteilt werden.

Der kurze $a$-Laut nach einem unmouillierten Konsonanten tritt je nach der Beschaffenheit des folgenden Konsonanten in jedem Dialekt in zwei Gestalten auf: wenn der folgende Konsonant unmouilliert ist, steht im Tundrajurakischen $a$ und im Waldjurakischen $a$, und wenn der folgende Konsonant 
mouilliert ist, steht im Tundrajurakischen $\underset{x}{a}$ und im Waldjurakischen $a$. Wir haben also z.B. O $\beta a \delta p$ aber P $\beta a t t p ~ ' H a k e n '$ und auf der anderen Seite von demselben Worte die Formen $O$ Badī Akk.Pl. und P Bătśíèj Nom.Pl. mit Possessivsuffix 1. P. Sing., ferner O màn' aber S maǹ 'Knäuel' 1 und auf der anderen Seite O mań aber $\mathrm{Nj}$ mań 'ich'. Aus dem O-Dialekt seien noch einige Beispiele angeführt: vor unmouilliertem Konsonanten

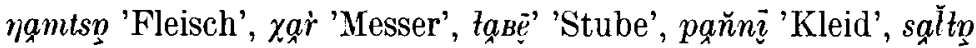
'dumm'; vor mouilliertem Konsonanten jadēr' 'Lichtung',

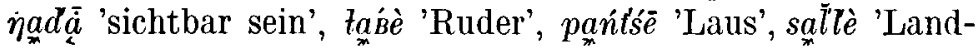
zunge'.

Der kurze $a$-Laut der ersten silbe nach einem mouillierten Konsonanten lautet vor einem unmouillierten Konsonanten in den meisten Dialekten $\dot{a}$, aber in OP nach $j$ gewöhnlich (doch nicht immer) $\varepsilon$. So haben wir $O$ jạaxà aber OP jexà 'Fluss',

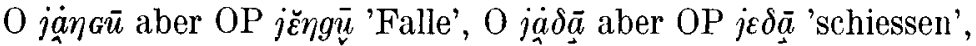

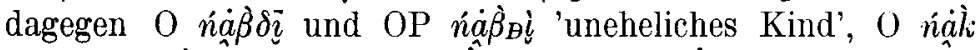

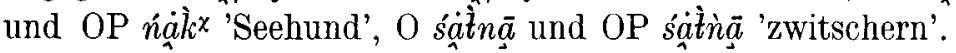

Der kurze $a$-Laut nach einem mouillierten Konsonanten in der Stellung vor einem mouillierten Konsonanten ist in recht wenigen Beispielen vorhanden (deren Zahl ist im WB nur etwa ein Dutzend). Nach $j$ scheint die Vertretung dieselbe wie vor unmouillierten Konsonanten zu sein, also in $\mathrm{O}$ (und den meisten anderen Dialekten) $\underset{a}{a}$ und in OP meistens $\varepsilon$, z.B. O jäl $\bar{a}_{\bar{c}}$ aber

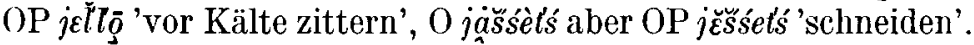
Sonst habe ich in $O$ und OP nur für die Stellung nach $n$ Beispiele gefunden. Auch hier hat $O P$ dieselbe Vertretung wie vor einem unmouillierten Konsonanten, also $\dot{a}$, in riăll $\bar{u}$ 'Unterrinde' und náämmui ' 'kurz und schlecht (von Renntierflechte)', aber $O$ hat in dieser Stellung ä, z.B. nä̆lle 'die Haare abwerfen', nä̈mmía 'hart sein', nä̈lt'śèt's' 'ausziehen (die Kleider)'. Auch die meisten anderen Dialekte haben $\ddot{a}$ in dieser Stellung. $\mathrm{T}_{2}$ hat $\dot{a}$ in sáăjem 'Seil'. OP hat $\underset{a}{a}$ in räämsitĭlì̀ 'schön machen', wo die Konsonantenverbindung $\hat{m} \hat{s}-\hat{-}$ wohl als mouilliert zu betrachten ist.

1 Das mit $\mathbf{S}$ bezeichnete Idiom wird in dem Verzeichnis der Sprachmeister nicht erwähnt, es vertritt aber offenbar einen waldjurakischen Dialekt, und zwar wohl wie in MSFOu 56 die Mundart von Sahalinskaja. 
Die (Wualität des langen (wie auch des halblangen) $a$-Lautes ist nach unmouillierten Konsonanten $a$, wenn der folgende Konsonant unmouilliert ist, und a, wenn der folgende Konsonant mouilliert ist. Die Vertretung in den verschiedenen Dialekten ist sehr einheitlich. Als Beispiele für die Stellung vor einem unmouillierten Konsonanten erwähnen wir $\mathrm{O} \eta \bar{a} \bar{\beta} \beta \bar{a} \delta$ 'Gürtelgegend', $\beta \bar{a} r$ 'Seite', pǟtti 'Schwert', $t \bar{a} \bar{\beta} \beta p$ 'Speck auf dem Bauch'. Die Stellung vor einem mouillierten Konsonanten haben wir z.B. in O $\eta \bar{a} j$ 'Tauwetter', $\eta \bar{a} j$ jo 'Körper', $\beta a \bar{a} u$ 'schneefreie Stelle', tàl b’̀̀ 'Felsenschlucht', tạś 'geben'.

In der Stellung nach einem mouillierten Konsonanten hat der lange $a$-Laut die Qualität $\dot{a}$ vor unmouilliertem und $\dot{a}$ vor mouilliertem Konsonanten. Wir erwähnen beispielsweise auf

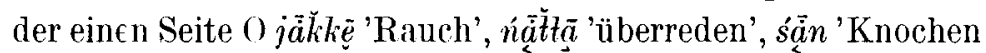
am Halfter', t'án̆nā 'hervorspritzen', auf der anderen Seite O

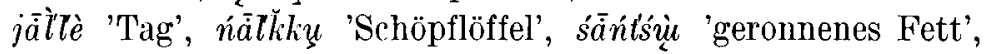
tännúu 'wenig'. Jedoch tritt auch vor einem unmouillierten Konsonanten nach labialen Konsonanten meistens die Qualität

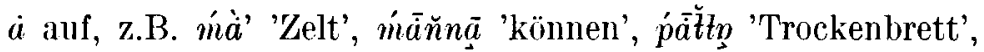
Wir haben sogar ä in f́ärńé 'entzwei schneiden', wo die Konsonintenverbindung -rn'-in ihrer Gesamtheit wohl als mouilliert zu betrachten ist (wie auch sonst ein unmittelbar vor einem mouillierten Konsonanten stehender Konsonant manchmal die Wirkung eines mouillierten Konsonanten ausübt, obgleich er nicht mit dem Mouillierungszeichen versehen ist).

Es sei hier eingeschoben, dass die Vokale im Auslaut dieselbe Qualität wie vor einem unmouillierten Konsonanten haben, z.B. $\eta \bar{a}$ 'Totengeist', $j \bar{a}$ 'Land', ńá 'Kamerad' (einsilbige Wörter mit auslautendem Vokal sind langvokalisch).

Die meisten jurakischen Dialekte haben bekanntlich keinen vokalischen Anlaut ausser in vereinzelten Interjektionen und jungen Lehnwörtern. In denjenigen Dialekten aber, die den sekundären anlautenden Nasal nicht haben, und auch sonst, wo ein anlautender Vokal vorkommt, hat er dieselbe Qualität wie nach einem unmouillierten Konsonanten, z.B. O äršsin (< russ.). Vergleiche weiter z.B. O namtsp $\sim$ U ampzp 'Fleisch', U $\eta \bar{a} j \sim$ Sjo $\bar{a} j$ 'Tauwetter'.

Der $o$-Laut scheint keinen phonologisch relevanten Unter- 
schied zwischen verschiedenen Längestufen zu haben. Er tritt meistens lang auf, bisweilen aber halblang und selten kurz. Um die Verteilung der Quantitätsstufen zu untersuchen, wollen wir als Probe den 0 -Laut der ersten Silbe in dem Idiom O bei Lehtisalo betrachten, und wir können im WB folgende Beobachtungen machen:

Die kurze Quantität des $o$-Lautes scheint hauptsächlich vor $\chi$ aufzutreten. Davon habe ich folgende Beispiele gefunden:

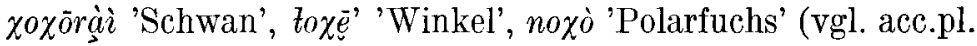

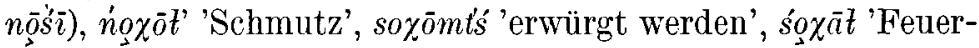

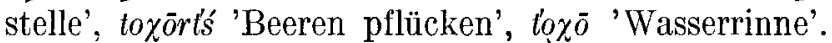

Vor einem anderen Konsonanten steht ein kurzes $o$ in den folgenden drei Beispielen: pottòttă 'schwatzen' (< russ.), poròmbā 'eilen' (aber OP, Sjo, Sj parr-), podórōmtāa 'starren' (aber Nj pat-, Sjo pad-). Unter dem Stichwort poròm Bă $_{x}$ steht u.a. O paró 'Eile haben'. In diesen drei Wörtern handelt es sich vermutlich eigentlich um ein kurzes $a$, das unter dem labialisierenden Einfluss des anlautenden $p$ und des o-Lantes der folgenden Silbe in o übergegangen ist. Wenn zwischen der ersten und der zweiten Silbe ein mouillierter Konsonant steht (wie in parōo), tritt die Labialisierung nicht ein.

Auch die halblange Quantität des o-Lautes steht in der Mehrzahl der Fälle vor $\chi$ (in 12 Fällen von 21): $\eta \dot{\alpha} \chi \bar{o}$ 'Kropf',

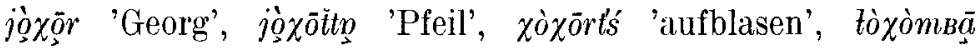

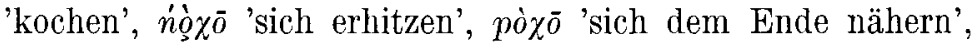

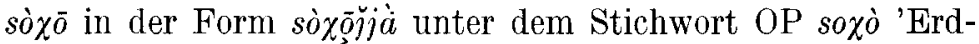
hügel', sò $\overline{\bar{o}}$ 'Biegsamkeit', tò $\chi \bar{e}$ ' 'Kattun', tò $\chi \bar{o}$ 'verschneit werden', tò $\chi \bar{o}$ 'lernen'.

Der halblange $o$-Laut steht ferner in 5 Fällen vor einem diphthongbildenden $i$ und in 2 Fällen vor $\dot{n}$. Diese Beispiele

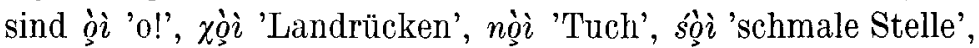
sóìdart'ś 'den Schlucken haben'; jọntèttità 'fortwährend lachen', sóñntśsè 'Höhle'.

Ausserdem haben wir pò̀ 'Pope' und tòmb'è 'flink', und schliesslich $\chi \grave{o} t \bar{k} k \bar{a}$ oder $\chi \bar{o} t \bar{k} k \bar{a} \vec{s}$ 'tauen'.

In der Stellung vor $\chi$ finden wir im O-Idiom unter den Stich-

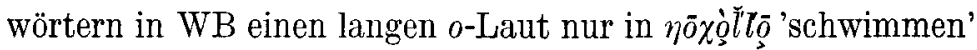
und jō $\chi \grave{o} t$ 'getrockneter Fisch' und in der Interjektion $j \bar{\partial} \chi \bar{o}$ 
'hoho!'. In der Stellung vor einem diphthongbildenden $i$ finden wir kein Beispiel von langem $o$-Laut in der ersten Silbe.

Der $o$-Laut der ersten Silbe tritt also normal als lang auf, scheint aber eine Tendenz zu haben, sich vor einem diphthongbildenden $i$ zur Halblänge und vor $\chi$ zur Halblänge oder Kürze zu verkürzen. Auch in anderen Stellungen kann ein halblanger oder kurzer $o$-Laut sporadisch auftreteten.

Die Qualität des o-Lautes ist von der Quantität unabhängig, was wenigstens nicht der Annahme widerspricht, dass die verschiedenen Längestufen dieses Lautes keine phonologische Relevanz haben. Nach einem unmouillierten Konsonanten steht vor einem unmouillierten Konsonanten und im Auslaut $o$, in den übrigen Stellungen aber, also nach unmouilliertem aber vor mouilliertem Konsonanten und immer nach mouilliertem Konsonanten, steht $o$, was von den folgenden Beispielen illustriert wird: einerseits $\eta \bar{o}$ 'Insel', $\eta \bar{o} \not{k} k \underline{\underline{x}}$ 'viel', mō̆nnāa 'fallen', tōrōmt's 'sich gewöhnen', andererseits aber $\eta \bar{o} d \grave{e}$ 'Beere', mōos 'werfen', sōjją 'verschaffen', jō' 'Ecke', śō 'Speiseröhre', tợr 'Ruf', jōtśs 'verlieren', tōnnne 'Fuchs'.

Die obenerwähnte Schlussfolgerung betreffs der phonologrischen Belanglosigkeit der verschiedenen Längestufen des $o$-Lautes wird gewissermassen davon unterstützt, dass die anderen halboffenen Vokale ein ähnliches Bild aufweisen. Diese Vokale sind $\bar{e}$ und $e$. Über deren gegenseitiges phonologisches Verhältnis wird unten gesprochen. Wir wollen sie vorläufig als zwei verschiedene, von einander unabhängige Vokale betrachten, so wie man es bisher gewöhnlich getan hat, und können dann feststellen, dass der zweite halboffene hintere Vokal, das illabiale $\ell$, überhaupt kaum als kurz auftritt. Nach einem labialen Konsonanten hat das $\underline{e}$ einen labialen Vorschlag und wird also diphthongisch realisiert, in der Gestalt $\underset{e}{e}$, wobei der halblange illabiale Vokal zusammen mit der überkurzen labialen Komponente als ein langer Vokal betrachtet werden

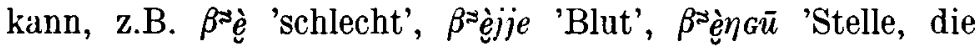
niemand aufsucht', mèptsamtā 'täuschen', $p^{\approx}$ ’èptā 'wickeln', oder in der Gestalt «è (so gewöhnlich nach $m$ ), z.B. $m \approx \grave{e} j$ 'Kraft, $m$ sèr $\bar{a}$ 'treffen'. In vereinzelten Fällen kann die labiale Komponente fehlen: Bètrp 'Eimer, Vedro', Bè̀mniè 'Sauerampfer', 
$p \grave{e} \beta \delta \bar{a}$ 'härten'. Sonst ist das $\underline{e}$ fast immer lang, wie z.B. in

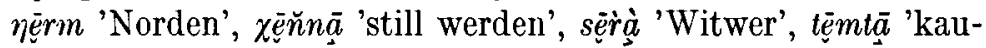
fen'. Ein kurzes $e$ habe ich unter den O-Wörtern nur in den beiden gleichlautenden Wörtern sexé 'menschenscheu' und sexē 'Aushau' (vor $\chi$ !) sowie in der Interjektion $\dot{\beta}_{\underline{e}}$ gefunden.

Der dritte halboffene Vokal, also $e$, tritt in der ersten Silbe der $\mathrm{O}$-Wörter in drei Fällen vor $\chi$ als kurz auf, nämlich

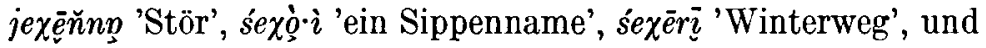
ausserdem ist er kurz in den beiden Wörtern jelte 'Weibchen' und sejejnnā 'schnell fliessen' (dasselbe Wort wie sèjnāa 'laut rasseln'?). Das halblange $e$ kommt verhältnismässig häufig vor, nämlich in den folgenden 27 Wörtern: jëi 'Anteil', jèi' 'weiche Stelle in der Seite', jèxèrā 'nicht wissen', jèmńą 'flicken', jèmt'sedèi 'Preiselbeere' (vgl. jēnt'sedèi id.), jèdàt 'Strähne', lèmbè 'Ruderblatt', lèmtērt's' 'streicheln', mèyò rrttā 'dick sein', mèrt'sè

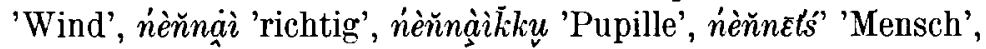
nèntsy 'gerade', nèntśêt' 'Möglichkeit', nèntśét's 'frei umhergehen', ’’’’ 'hohle Hand', p’è $\beta$ 'innere Rinde der Weide', ṕè̀è $\delta a \bar{a}$ 'sich plötzlich öffnen', ṕè̀è $\delta \bar{a}$ 'krachen, knallen' (die zwei letztgenannten Wörter dürften in der Tat ein und dasselbe Wort darstellen, und das nächste Wort ist wohl eine Ableitung desselben Grundstammes), ṕè $\bar{e} \cdot r \grave{c} t \bar{a}$ 'fast nach hinten aufmachen, nach hinten aufbrechen', seìi 'Herz', śèjnā 'laut rasseln', séx̀̀ 'harter Schnee', śè èdạ 'herausfallen und verschüttet werden', śèmṕè 'gehen, ohne einzusinken', tè̆pā̄n 'Stephan'. In der weit überwiegenden Mehrzahl der Fälle (etwa 170) ist indessen $e$ lang, und wenn wir die lange und die halblange Stufe zusammen der kurzen gegenüberstellen, scheint es völlig sicher, dass man auch bei $e$ keine phonologisch relevanten Quantitätsgegensätze anzunehmen hat.

Bei den engen Vokalen $i, \underline{i}$ und $u$ muss man wohl dagegen, ebenso wie bei dem offenen Vokal $a$, mit zwei phonologisch relevanten Quantitätsstufen rechnen. Bei a kann man leicht solche Gegensätze finden wie $0 \beta a g \underline{x}$ 'Haken' $\sim \beta \vec{a} \delta \underline{p}$ 'Wort', $s a \tilde{\beta} \beta \underline{\underline{p}}$ 'gut' $\sim s \bar{a} \bar{\beta} \beta \underline{\underline{p}}$ 'Hochwasser'. Bei den engen Vokalen ist es nicht so leicht, völlig so klare Fälle zu finden, aber z.B. die folgenden sind wohl recht überzeugend: O pidè 'Nest' $\sim$ pīdēe

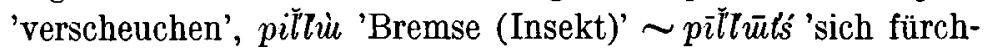


ten', tïr' 'Kante' $\sim$ tìr 'Wolke', sirrrp̣ 'Schnee' $\sim$ sīrā 'sich schämen', pur̀' 'Mückenfeuer' pūr' 'Rost'.

Wir kommen also zu dem Ergebnis, dass in dem jurakischen Vokalsystem die offenen und engen Vokale zwei Quantitätsstufen, die lange und die kurze, haben, während dieser Gegensatz bei den halboffenen Vokalen fehlt. Vergleiche Steinitz, Geschichte des finnisch-ugrischen Vokalismus, Seite 132-133: "Die Vokalqualitäten der heutigen jur. Dialekte und des Urjur. sind somit $\underset{u}{o} \underset{i}{e} e_{i}^{e}$. Alle diese Vokale kommen la ng (Leht. $\bar{a}$ usw.) vor; als $\mathrm{k}$ u z e sind weite und enge $(a, u, \underline{i}, i)$ sicher belegt, betreffs der mittelweiten erlaube ich mir rorläufig kein (Trteil."

Die Vokale $e, e, i, i$ haben dieselbe Qualität vor mouillierten wie vor unmouillierten Konsonanten, was aus den oben gegrebenen Beispielen zur Genüge hervorgehen dürfte. Auf das gegenseitige Verhältnis zwischen $e$ und $\underline{e}$ bezw. $i$ und $\underset{i}{ }$, das heisst ihre Abhängigkeit von der mouillierten oder unmouillierten Beschaffenheit des vorhergehenden Konsonanten, werden wir etwas später zurückkommen.

Wir haben schon festgestellt, dass der o-Laut die beiden Varianten $o$ und $g$ hat, von denen $g$ in solchen Fällen steht, wo vor oder nach dem Vokal ein mouillierter Konsonant steht, während $o$ in unmovillierter Umgebung auftritt. Es bleibt uns jetzt übrig, die qualitativen Verhältnisse des $u$-Lautes zu untersuchen.

Der $u$-Laut hat fünf qualitative Varianten, nämlich $u$, $\iota$, $u$, $u$ und in drei Wörtern der O-Mundar tü. In unmouillierter Umgebung, also wenn sowohl der vorhergehende als der nachfolgende Konsonant unmouilliert ist (oder wenn der Vokal nach unmouilliertem Konsonanten im Auslaut steht), wird die Variante $u$ verwendet, z.B. $\eta u \grave{r} r \bar{z} B \bar{a}$ 'schlagen', $\chi u r t s \bar{a}$ 'lärmen',

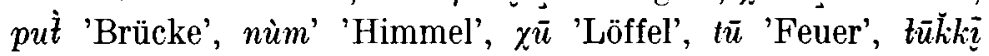
'Klumpfpfeil'. (110 Belege.)

Wenn der $u$-Laut nach unmouilliertem aber vor mouilliertem Konsonanten steht, hat er die Qualität $u$, z.B. $p u j$ 'eine Art

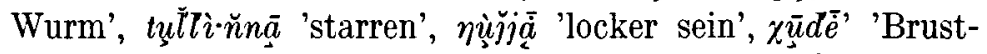
bein des Vogels', nụ̂s 'stehen', pựt's 'blasen', tụ̄ñni 'Flinte'. 
(Die Zahl der Belege ist 34.) Es ist zu merken, dass hier wie sonst ein diphthongbildendes $i$ als ein mouillierter Konsonant gerechnet wird (z.B. pưi 'hinten befindlich', Buirkkp 'Filz'), und ebenso, dass ich hier und anderswo in dieser Darstellung unter die Fälle mit mouilliertem Konsonanten diejenigen aufnehme, wo ein mit Mouillierungszeichen nicht versehener Konsonant vor $e$ oder $i$ steht, falls er sonst dieselbe Qualität wie der fragliche mouillierte Konsonant hat (von dem Grunde dazu wird unten gesprochen). Drei Wörter dieser Art sind bei dem jetzt behandelten Punkte aktuell, nämlich $\chi_{\mathcal{u}}^{\bar{u}} \bar{n} n \bar{\imath}$ ' 'dicke Zelt-

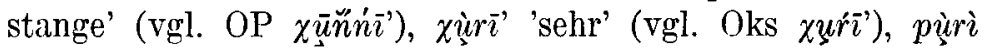
'Hecht' (vgl. OP purí).

Nach unmouillierten Konsonanten ist also das Grundzeichen $u$, und wenn der Vokal vor einem mouillierten Konsonanten steht, wird die Variante $u$ verwendet. Nach mouillierten Konsonanten ist das Grundzeichen $u$, das die Variante $u$ hat, und ausserdem kommt in sehr wenigen Fällen $\ddot{u}$ vor. Die Verteilung ist hier beim ersten Anblick nicht so klar wie nach einem unmouillierten Konsonanten. Vor einem unmouillierten Konsonanten (oder im Auslaut) steht $u$ in 50 Fällen und $u$ in 14 Fällen. In der Stellung nach einem unmouillierten Konsonanten spielt, wie aus den obigen Beispielen ersichtlich ist, die Quantität keine Rolle, aber in der Stellung nach einem mouillierten Konsonanten muss sie offenbar in Betracht gezogen werden. Es stellt sich nämlich heraus, dass von den genannten 50 Fällen der $u$-Variante 45 lang (oder halblang)

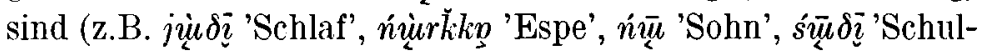
terblatt'), während nur in den folgenden fünf Wörtern das $u$ kurz ist: jux $\bar{u}$ 'Netzmagen', jum 'schmale Flussbucht', jùn 'Kenntnis', jư̆ng 'Pferd', jun̆nù 'Frihling'. Es ist zu beachten, dass diese fünf Wörter im Anlaut $j$ haben. Beispiele für einen langen (oder halblangen) $u$-Laut in der fraglichen Stellung, die eine andere Variante als $u$ hätten, gibt es nicht ausser dem Wort pùusart's 'aus dem Mund spritzen', das in den anderen Dialekten (OP, Sjo, $\mathrm{Nj}, \mathrm{T}_{1}$ ) mit kurzem Vokal der ersten Silbe aufgezeichnet ist und vielleicht in der Wirklichkeit zur nächsten Gruppe gehört. Wir können also vorläufig die Regel aufstellen, dass nach einem mouillierten Kon- 
sonanten der lange $u$-Laut vor unmouilliertem Konsonanten die Qualität $\psi$ hat.

Wir haben soeben gesehen, dass es fünf Wörter gibt, in denen der kurze $u$-Laut in der fraglichen Stellung von der Variante $u$ vertreten ist, und dass diese Wörter mit $j$ anfangen. Auch das Wort juxō 'verschwinden' hat im Anlaut $j$ aber trotzdem die Vokalvariante $u$. Wenn man die Regel aufstellen will, das die mit $j$ anlautenden kurzvokaligen Wörter in dieser Stellung $u$ haben, bleibt also dieser Fall eine Ausnahme. Sonst gibt es hier 12 Fälle von kurzem $u$-Laut, und sie haben alle

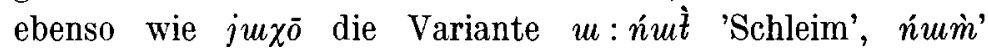

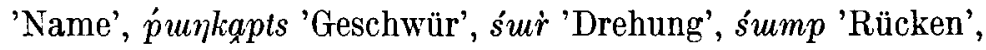
śumвап

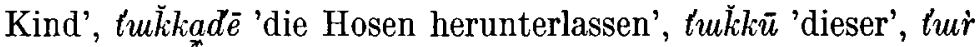
'Treibstock', 'tunt 'Landrücken'.

Da also auf diesem Punkte eine gewisse Unklarheit besteht, wollen wir ausnahmsweise das sonst in dieser Darstellung verwendete Material, also Lehtisalos Wörterbuch, verlassen und statt dessen seine Juraksamojedische Volksdichtung (MSFOu 90) heranziehen, und es stellt sich dann heraus, dass in den Texten, die nach demselben Gewährsmann wie die O-Wörter des WB aufgezeichnet sind, der kurze $u$-Laut in der fraglichen Stellung - nach mouilliertem aber vor unmouilliertem Konsonanten - auch in den mit $j$ anlautenden Wörtern als $u$ aufgezeichnet ist, z.B. Seite 537, Zeile 9 (nach der Marginal-

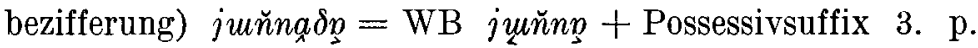
sing. Wir wollen deshalb bei der Formulierung der Regeln die obigen fünf Wörter mit kurzem $\psi_{\llcorner}$ausser Acht lassen und $u$ als die regelrechte Aussprache betrachten. Man vergleiche jedoch, dass der kurze a-Laut vor mouillierten Konsonanten nach $j$ eine analoge Sondervertretung hat (nicht $\ddot{a}$ sondern $\underset{a}{a}$ ).

Wenn der $u$-Laut lang oder halblang ist und zwischen zwei mouillierten Konsonanten steht, hat er ebenfalls die Qualität $u$. Hiervon gibt es 21 Beispiele, wobei freilich in ein paar Fällen der nach dem $u$-Laut stehende Konsonant ein Beispiel von der oben kurz angedeuteten und unten näher zu besprechenden "potentiellen Mouillierung" vor $i$ oder $e$ darstellt, d.h. der Konsonant ist nicht als mouilliert bezeichnet aber fungiert in jeder Hinsicht als ein mouillierter Konsonant. Die 21 Wörter 
sind die folgenden: jürìik 'getrockneter Fisch', jürúu 'Freund', jưssi $i \bar{a}$ 'liegen', jùtts' 'in Stücke hauen', jùté 'mit verlorenen

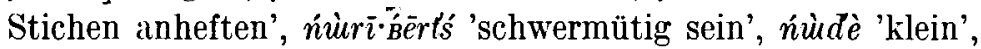
tuiunńne 'Feuer anmachen', jǖjjemts's 'aufspringen', jūus' 'schmel-

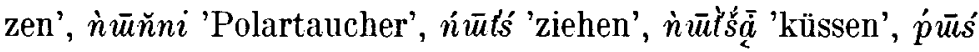

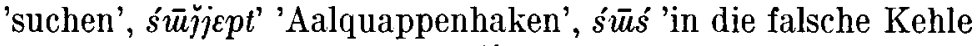
geraten', tūulliśé 'ein Vogel', tümìù 'Eisenblech der Feuerstelle', 'tūss 'hineingehen', t'ūsssser' 'Decke auf der Windseite der Rauchöffnung', und schliesslich śùlt'ś 'das Rieseln'. In dem letzten Beispiel soll das $l$ als zur Gruppe der "mouillierten Konsonanten" gehörig betrachtet werden (durch die Einwirkung des nachfolgenden t's, vgl. s.v. Kis šutttaš 'rieseln' die Wörter O śúutnä 'fortwährend laufen (Flüssigkeit aus etwas)' und śųlttarity 'kleiner Bach') in derselben Weise wie die obenerwähnten "potentiell mouillierten" Konsonanten vor $i$ und $e$, denn sonst würde hier $t$ und nicht $l$ stehen.

Wir kommen schliesslich zu der Variante $\ddot{u}$ und müssen trotz der äusserst grossen Knappheit des Materials schliessen, dass sie die Vertretung des kurzen $u$-Lautes zwischen zwei mouillierten Konsonanten ist (vergleiche die Vertretung des kurzen $a$-Lautes durch $\ddot{a}$ in dieser Stellung). Das $\ddot{u}$ tritt im O-Dialekt in drei Belegen auf: pül'se 'nasse Stelle im Moor, in die man tief einsinkt', püri'ènGōos' 'Störstange', tüñné é 'riechen'. Das erste dieser Wörter wird von Lehtisalo unter unmouilliertes $p$ aufgenommen und heisst in $\mathrm{OP}$ pulbe (in $\mathrm{T}_{1}$ und $\mathrm{T}_{5}$ dagegen mit $\ddot{u}$ ), in unserem Dialekt aber kann das $p$ vielleicht als "potentiell mouilliert" betrachtet werden (vergleiche die unten zu behandelnde Tatsache, dass das "nouillierte $p$ " vor dem anderen engen Vordervokal $i$ immer ohne Mouillierungszeichen auftritt). Das Wort kann übrigens onomatopoetisch sein. Auch das zweite Wort hat im Anlaut $p$ ohne Mouillierungszeichen, obschon es von Lehtisalo unter $p$ steht, und auch das $r$ ist ebenso wie das oben behandelte $l$ in śült's als zu der Gruppe der "mouillierten Konsonanten" gehörig zu betrachten, obschon dies bei $r$ nicht wie bei $l$ aus der Qualität des Konsonanten hervorgeht. Das Wort tiünñe ist somit das einzige in jeder Beziehung ganz einwandfreie Beispiel für den kurzen $u$-Laut zwischen zwei mouillierten Konsonanten.

Nach dieser ziemlich langen Auseinandersetzung können wir 
die Regeln für den $u$-Laut der ersten Silbe in der Stellung nach einem mouillierten Konsonanten im O-Dialekt folgendermassen zusammenfassen:

1. Die lange Quantität hat vor ummouilliertem Konsonanten die Qualität $u$.

2. Die kurze Quantität hat vor unmouilliertem Konsonanten die Qualität $u$.

3. Die lange Quantität hat vor mouilliertem Konsonanten die Qualität $u$.

4. Die kurze Quantität hat vor mouilliertem Konsonanten die Qualität $\ddot{u}$.

Unter der langen Quantität ist hier wie sonst die halblange eingerechnet.

Man kann die interessante Feststellung machen, dass del kurze Vokal etwas mehr nach vorn artikuliert wird als der lange Vokal in derselben Stellung, was darin resultiert, dass der kurze Vokal vor unmouilliertem Konsonanten dieselbe (qualität hat wie der lange Vokal vor mouilliertem Konsonanten. Dies gilt nur für die Stellung nach einem mouillierten Konsonanten, in der Stellung nach einem unmouillierten Konsonanten dagegen ist die Quantität für die Qualität gleichzültig, wie wir oben gefunden haben. Auch bei dem a-Laute sind die Verhältnisse ähnlich, wie aus der Behandlung dieses Vokals am Anfang des Aufsatzes hervorgeht. Auch dort haben die verschiedenen Längestufen in der Stellung nach eimem unmouillierten Konsonanten keine Bedeutung für die Qualität, wenn man die horizontale Artikulationslage betrachtet, und der einzige Unterschied zwischen dem kurzen und dem langen Vokal ist derjenige, dass kurzes $a$ etwas enger artikuliert wird als langes $a$ in derselben Stellung: $\bar{a} \sim a$ vor unmouilliertem und $\bar{a} \sim a$ vor mouilliertem Konsonanten. Wenn wir von diesem Unterschied in der vertikalen Artikulation absehen, sind die Verhältnisse auch nach mouillierten Konsonanten dieselben bei dem $a$-Laut wie bei dem $u$-Laut: den Zusammenstellungen $\bar{u} \sim u$ vor unmouilliertem und $\bar{u} \sim \ddot{u}$ vor mouilliertem Konsonanten entspricht $\overrightarrow{\dot{a}} \sim \dot{a}$ bzw. $\bar{a} \sim \ddot{a}$.

Wenn man die soeben beschriebene Erscheinung in einer allgemeiner gefassten Regel formulieren will, könnte sie folgendermassen lauten: Bei denjenigen Vokalen, die phonologisch 
zwei Längestufen haben und die in der Stellung nach einem mouillierten Konsonanten überhaupt verschiedene qualitative Varianten habell, wird nach einem mouillierten Konsonanten die kurze Quantität mehr palatal artikuliert als die lange Quantität in derselben Stellung, wobei die kurze Quantität vor unmouilliertem Konsonanten denselben Grad von Palatalität bekommt wie die lange Quantität vor mouilliertem Konsonanten; in der Stellung nach einem unmouillierten Konsonanten ist die Quantität bei sämtlichen Vokalen für die horizontale Artikulation belanglos.

Über das Quantitätssystem müssen noch einige Worte gesagt werden. Ich habe in der obigen Darstellung im allgemeinen angenommen, dass die langen und die halblangen Vokale (sowie natürlich die vereinzelt vorkommenden Fälle von überlangem Vokal, z.B. in der Interjektion $\hat{u}$ ) phonologisch betrachtet zusammen eine Quantitätsstufe bilden, die lange, welcher dann ausser bei den halboffenen Vokalen die kurze Stufe entgegenzustellen ist. Es muss aber beachtet werden, dass in den Aufzeichnungen Schwankungen der Quantität bei einem und demselben Wort vorkommen, besonders interdialektal aber auch innerhalb der einzelnen Idiome, worauf hie und da in der obigen Darstellung auch hingewiesen wurde. Solche Schwankungen können natürlich bisweilen von dem Sprechtempo, dem Grad von Betonung und anderen gelegentlichen Faktoren abhängen. Lehtisalo schreibt im Vorwort zu seiner Textsammlung "Juraksamojedische Volksdichtung" (MSFOu 90, S. XI): "Und da die Quantität der langen Vokale im Tundrajurakischen kürzer ist als z.B. im Finnischen, verschwimmt beim flüchtigen Sprechen oft die Grenze zwischen den langen und halblangen Vokalen. Ebenso können bei schneller Sprechweise die halblangen Vokale den kurzen nahe kommen, und die Tundrajuraken sprechen im allgemeinen ziemlich schnell, bedeutend flüchtiger als z.B. die Waldjuraken. Die langen Schlussvokale waren bei einigen Gewährsleuten bisweilen sogar zu kurzen reduziert, während umgekehrt der Schlussvokal gelegentlich durch Abtönung zu einem langen gedehnt werden konnte.» Diese Verhältnisse verdienen jedenfalls, näher untersucht zu werden, was aber diesmal zu weit führen würde. Es sei nur hervorgehoben, dass die Qualität der Vokale so gut wie immer 
mit der in jedem einzelnen Fall tatsächlich vorliegenden Quantität in Einklang steht. Wenn also ein kurzer Vokal gelegentlich aus irgendeinem Grunde zur Halblänge gedehnt wird, so bekommt er die bei der langen Stufe zu erwartende Qualität, und umgekehrt. Als ein Ausnahmebeispiel könnte man 0 náâjjèlt's 'mahlen' (mit interdialektaler Schwankung zwischen kurzem, halbkurzem und langem Vokal der ersten Silbe) erwähnen, das trotz dem halblangen Vokal die bei kurzem $a$-Laut zwischen zwei mouillierten Konsonanten zu erwartende Qualität $\ddot{a}$ hat.

Was die zufälligen, phonologisch und morphologisch bedeutungslosen Schwankungen zwischen der langen und der halblangen Quantität im Auslaut betrifft, die z.B. im Nom.Sg. und Akk.Pl. gewisser Worttypen auftreten, so hat schon Hajdú (a.A., S. 249) dargelegt, dass nicht ich bei der Beurteilung dieser Erscheinung "auf Irrwege geraten» bin, wie Joki (a.A., S. 19) behauptet, sondern dass im Gegenteil Joki dies getan hat. Es ist vergebliche Mühe, solche in den Aufzeichnungen vorkommende phonetische Gegensätze zwischen Nom.Sg. und Akk.Pl. wie einerseits $t \grave{u} \chi \grave{u} \sim t \grave{u} \chi \bar{u}$ und andererseits $\dot{n} \bar{u} \sim \dot{n} \dot{u}$ mit Joki morphologisch systematisieren zu wollen. Die im Akk.Pl. häufigen auslautenden $u$ - und $i$-Laute sind phonologisch immer lang, können aber phonetisch halblang sein. In den soeben angeführten Beispielen hat der zweisilbige Akk.Pl. langen Auslaut, der einsilbige dagegen halblangen. Man kann aber auch das umgekehrte Verhältnis finden, z.B. (nach WB) OP Nom.Sg. $t \underline{i} \sim$ Akk.Pl. $t \underline{i}$, Nom.Sg. narirrì $\sim$ Akk.Pl. (in einem Satzbeispiel) narrrù. Und der in demselben Satz vorkommende Akk.Pl. OP $\beta \bar{i} \eta \grave{u}$ hat unter dem Stichwort $\beta \bar{\imath}$ ' 'Moor' in $O$ die Form $\beta \bar{z} \eta \bar{u}$. Dagegen will ich nicht verneinen, dass ein phonologisch relevanter Wechsel zwischen $\mathrm{k} \mathrm{u} \mathrm{r} \mathrm{z} \mathrm{e} \mathrm{m}$ Auslaut im Nom.Sg. und langem im Akk. Pl.vorkommt, und Joki kann insofern recht haben, als der Nom.Sg. der einschlägigen Worttypen nicht i m m e r mit dem Akk.Pl. identisch ist. In solchen Fällen ist der lange Auslautsvokal des Akk.Pl. meines Erachtens auf die ursprüngliche Endung * $*_{-i}$ zurückzuführen. 
Nach den Regeln, die von Prokof'ev (Jazyki i pis'mennost' narodov severa, I, S. 12) für die Aussprache und Schreibung des Jurakischen gegeben werden, "sind vor den Vokalen $i$ und $e$ die Konsonanten in allen Fällen ohne Ausnahme mouilliert, in der Schrift aber werden die Konsonanten vor $i$ und $e$ ohne das Mouillierungszeichen ausgedrückt, z.B. sida 'zwei' (statt śida), ni 'Gürtel' (statt ńi) usw.» Dieselbe Schreibweise haben Collinder und ich verwendet, Joki aber sagt in seiner Besprechung (FUF 32, Anzeiger, S. 6) meiner Abhandlung "The form of the object in the Uralic languages" (im Folgenden FO verkürzt), und auch in seiner Besprechung (ib., S. 43) von Collinders "Fenno-Ugric vocabulary", dass dieses Verfahren unzulässlich sei, da vor $i$ und $e$ auch unmouillierte Konsonanten stehen können. Es sei deshalb notwendig, auch vor $i$ und $e$ das Mouillierungszeichen bei den Konsonanten zu setzen, und Joki gibt einige Beispiele von einem unmouillierten Konsonanten vor $i$. Die selkupischen Beispiele, die er anführt, haben indessen in diesem Zusammenhange nichts zu tun, denn weder Collinder noch ich haben behauptet, dass die einschlägige Regel auch für das Selkupische Geltung habe. Was das Jurakische betrifft, sagt Joki, das gewöhnliche jurakische Verb für 'geben' heisse $m i$ - und dürfe nicht $m i$ - gelesen werden. Indessen hat Lehtisalo in seinem Wörterbuch (das damals allerdings noch nicht erschienen war) dieses Wort unter $\dot{m}$ und nicht unter $m$, und er gibt für den Infinitiv die folgenden Dialektformen: $\mathrm{O}$ mit's, $\mathrm{Sj}, \mathrm{K}$ mitś, $\mathrm{Nj}$ miś. Eine Besichtigung des Materiales im WB gibt an die Hand, dass das Mouillierungszeichen recht oft und anscheinend regellos vor $i$ und $e$ fehlt, und Lehtisalo selbst kommentiert dieses Verhältnis im Vorwort des WB folgendermassen (S. CVII): »In gewissen Dialekten oder bei einigen Sprachmeistern des Tundrajurakischen war die Mouillierung im Zusammenhang mit einigen Konsonanten vor $i$, $e$ nicht zu hören, aber z.B. $t, d$ klangen immer deutlich mouilliert. Vor anderen Vokalen ist die Mouillierung immer deutlich zu hören. In der eilig aufgezeichneten Folklore kann das Fehlen des Mouillierungszeichens auch davon herrühren, dass die Mouillierung beim schnellen Sprechen in solchen Fällen, wo man sie vor $i$ und $e$ erwartet hätte, 
unbemerkt geblieben ist." Lind in dem Vorwort zu seiner Textsammlung sagt er (MSFOu 9u, S. XI): "Erwähnung verdient ferner, dass in den tundrajurakischen Texten Schwankungen bei der Verwendung des Mouillierungszeichens einiger Konsonanten vor den $e$ - und $i$-Vokalen vorkommen. Im Tundrajurakischen konnte damals nämlich die Muuillierung, besonder's bei schnellem Sprechen, sekundär so reduziert sein, lisss ich sie oft unbezeichnet liess.»

Die oben angeführte Regel Prokof'evs bezieht sich aui dit damalige (1937) jurakische Schriftsprache, die mit dem lateinischen Alphabet geschrieben wurde. Dort wird die Mouillierung der Konsonanten vor $a, o, u$ mit dem Mouillierungszeichen angegeben, vor $i$ und $e$ dagegen nicht. In der heutigen jurakischen Schriftsprache, die mit dem russischen Alphabet geschrieben wird, wird nach dem Muster des Russischen die Mouillierung der Konsonanten vor einem Vokal durch das Vokalzeichen angegeben: vor den Vokalzeichen $я, \ddot{\mathbf{e}}$, ю, и, е sind die Konsonanten mouilliert, vor a, o, y, ы, э dagegen unmouilliert. Wenn ein Vokalzeichen der ersteren Reihe am Anfang eines Wortes oder nach einem Vokalzeichen steht, bedeutet dies, dass vor dem Vokallaut ein $j$ ausgesprochen wird, z.B. и' = Lehtisalo jī' 'Wasser', еся $=$ L. jëšse 'Eisen', яна $=\mathrm{L}$. jännn 'ruhig', ёр = L. jōr 'Tiefe'.

Ein Durchgang des WB-Materials zeigt, dass das Mouillierungszeichen viel häufiger vor $i$ als vor $e$ weggelassen wird. Wenn wir die anlautenden Konsonanten des O-Dialektes untersuchen, können wir folgende Feststellungen machen:

$l$ ist immer mouilliert vor $e$ (9 Belege) aber immer unmouilliert vor $i$ (6 Belege), z.B. lèmsè 'Ruderblatt' aber limb́è 'Adler' (vgl. z.B. $\mathrm{T}_{1}$ limbe aber $\mathrm{T}_{12}$ limìiè id.).

$m$ vor $e$ kommt in 10 Stichwörtern vor; es ist mouilliert in allen ausser mérú 'Wunde'. Doch steht unter diesem Stichwort (das in den meisten anderen Dialektformen $\dot{m}$ hat) auch das Verb 0 mérùu lēe 'verwunden'.

$m$ vor $i$ haben wir in 15 Wörtern; es ist unmouilliert in 10 und mouilliert in 5 Fällen. Es ist vielleicht kein blosser Zufall, dass in 9 von den 10 Wörtern mit unmouilliertem $m$ der nach $i$ stehende Konsonant auch unmouilliert ist, während er in 
sämtlichen $5 \dot{m}$-Fällen mouilliert ist (man vergleiche unten, dass dieselbe Tendenz bei $n$ zu spüren ist). Das unmouillierte $m$ haben wir in den Wörtern mǐkkì tài 'Nikolaus', mīr' 'Form', mirnā 'schnell gehen', mirt'śät'é 'plötzlich werfen', min 'Richtung', min̆nāa 'auf der Wanderung stehen bleiben', min̆naréñnn 'Fahrer', miñnat'ś 'schnell gehen', miskp jāllte 'Michaelisfest' und schliesslich (mit mouilliertem Konsonanten nach i) in miniekki 'die grosse Zehe'. Den mouillierten $m$-Laut haben wir in miñné 'tragen', minilè 'ein Geistervogel', minit's 'gehen', miśs 'machen', mit's 'geben'.

$n$ vor $e$ komnt reichlich vor (über 30 Belege), und es ist überall mouilliert.

$n$ vor $i$ haben wir in 24 Wörtern, in 9 Fällen unmouilliert und in 15 Fällen mouilliert. Der nach $i$ stehende Konsonant ist unmouilliert (oder, in einem Fall, das $i$ ist auslautend) in 7 von den 9 Fällen, und er ist mouilliert in 11 von den 15 Fällen. Den unmouillierten $n$-Laut haben wir in $n \bar{\imath}$ 'Gürtel', nit 'mindermassiger Fisch', nīr' 'Messergriff', nir $\delta$ 'Eisspross des Geweihs', nīn 'Seitenholz des Schlittenbodens', niñn $n^{a} r e \bar{t} t s$ 'schichtenweise sein', nīnme 'Oberfläche der Oberschenkel beim Sitzen', und mit dem folgenden Konsonanten mouilliert in niìèdż 'Mann der älteren Schwester', nišśè 'Vater'. Den mouillierten n-Laut finden wir in niňnéhkà 'älterer Bruder', niint'ši' 'Gaumen', níisè 'Nadel', nì Bèrị 'ein kleines Insekt, Thrips', niìi 'Spinne', niis' 'nicht', nits's 'Junge werfen' nithtì', 'unteres Ende des Pfeiles', niid'ét's' 'Vorderquerstange des Schlittens', ní 'Aussenseite eines krummen Baumes', und mit dem folgenden Konsonanten unmouilliert in 'ń' 'auf', ńiximts's 'verschlingen', ñîrt's' 'Augenbraue', nimńé 'saugen', nipka âttā 'vornübergebeugt sein'. Von den letzteren Wörtern sind ńïr's's und nimńée mit Bezug auf den nach $i$ folgenden Konsonanten nicht unzweideutig, da man wie schon erwähnt bei einigen Wörtern die Erscheinung beobachten kann, dass ein mit Houillierungszeichen nicht versehener Konsonant vor einem mouillierten Konsonanten kombinatorisch als ein mouillierter Konsonant fungiert, z.B. wenn der vor dem Konsonanten stehende Vokal nicht $\underset{\alpha}{a}$ sondern $\underset{x}{a}$ ist oder der Konsonant selbst nicht $t$ sondern $l$ ist. Auch ñizim t's stellt gewissermassen einen Sonderfall dar, 
da hier trotz dem unmouillierten Konsonanten $\chi$ die zweite Silbe den Vokal $i$ und nicht $i$ hat, was auf der assimilatorischen Regel beruht, dass nach $\chi$ derselbe Vokal wie vor ihm steht; mehr davon unten.

$p$ vor $e$ steht in 27 Wörtern, und es ist mouilliert in allen ausser pē̌lle 'Verwandter', pē̌llè 'Hälfte' (verschiedene Meinungen sind darüber ausgesprochen worden, ob diese zwei Wörter etymologisch identisch sind oder nicht), pēm̄me 'Zunder'.

$p$ vor $i$ wird in 35 Wörtern angetroffen, und zwar überall unmouilliert.

Nach der oben angeführten Angabe Lehtisalos ist $t$ vor $e$ und $i$ immer mouilliert, was von dem Material bestätigt wird. Dasselbe gilt auch für $s$.

Einige Tendenzen sind also recht klar zu erblicken. Vor $e$ sind die Konsonanten fast immer mouilliert, vor $i$ aber können sie sowohl mouilliert als unmouilliert sein, welcher phonetischer Unterschied keinerlei bedeutungsdifferenzierende Funktion hat. Besonders die labialen Konsonanten treten gern vor $i$ unmouilliert auf, die Dentalkonsonanten dagegen nur ausnahmsweise. Diese Tendenz ist am klarsten bei den Explosiven zu beobachten: $p$ ist im 0 -Dialekt im Anlaut vor $i$ immer unmouilliert, $t$ dagegen nie.

Die stets unmouillierten Konsonanten $\eta, \chi$ und (im Tundrajurakischen) $\beta$ stehen anlautend nie vor $e$ oder $i$, ebenso wie das nie unmouillierte $j$ nicht vor $\ell$ und $i$ stehen kann.

Wir haben also wenigstens einige Tendenzen zu einer gewissen Regelmässigkeit im Wechsel der mouillierten und unmouillierten Konsonanten vor $i$ und $e$ gefunden, und diese Tendenzen dürften einen phonetischen Grund haben. Die ganze Erscheinung ist ja phonetischer Art und hat keine morphologische oder sonstige semantische Bedeutung. Der wichtigste Zug in der Verteilung scheint mir derjenige zu sein, dass unmouillierte Konsonanten oft vor $i$ aber selten vor $e$ auftreten. Nun ist ja $i$ derjenige Vokal, der den ausgeprägtesten palatalen Charakter hat, da er neben der vorderen Zungenstellung auch die höchste Zungenstellung und deshalb die höchste Mundresonanz hat. Bei der Aussprache eines mouillierten Konsonanten bedeutet das Mouillierungsmoment eine $i$-ähnliche Artikulation und einen $i$-ähnlichen akustischen Effekt. Wenn die 
Mouillierungsartikulation schwach ist, kann die Mouillierung des Konsonanten und der Klang des folgenden $i$-Vokals für das $\mathrm{Ohr}$ leicht in einen mehr oder weniger undifferenzierten Eindruck zusammenschmelzen, und andererseits kamn es für denjenigen, der das Jurakische als seine Muttersprache verwendet, gleichgültig sein, ob die Mouillierung bei jeder Gelegenheit wirklich in ausgeprägter Form vorliegt oder nicht, da z.B. ein $m i$ nicht in einem Oppositionsverhältnis zu einem $m i$ stehen kann, sondern nur zu einem $m i$. Der Unterschied zwischen $m i$ und $m i$ kann von einem Gesichtspunkt so ausgedrückt werden, dass der Übergang zur $i$-Artikulation, d.h. die Hebung des Zungenrückens, im letzteren Falle etwas später stattfindet als im ersteren. In der Stellung vor $e$ dagegen liegt die Sache etwas anders. Hier ist der Unterschied zwischen der Mouillierungsartikulation und der $e$-Artikulation (und zwischen den entsprechenden akustischen Eindrücken) ausgeprägter. Die Zunge soll zuerst in die Lage der Mouillierungsartikulation gehoben und danach in die Lage der $e$-Artikulation gesenkt werden. Der Unterschied zwischen z.B. me und me wird deshalb ausgeprägter als derjenige zwischen $m i$ und $m i$, und er wird nicht so leicht verschwommen oder ausser Acht gelassen (und dies dürfte sowohl für die tatsächliche Aussprache gelten als für die Leichtigkeit des Aufzeichners, bei schnellem oder etwas undeutlichem Sprechen den Unterschied zu beobachten). Diese Verhältnisse können wohl erklären, warum wir in dem jurakischen Material eine ausgesprochen stärkere Tendenz zur Weglassung der Mouillierung vor $i$ als vor $e$ finden. Bei den Vokalen $a, o, u$ (ausser bei der Variante $\ddot{u}$ ) wiederum ist der Unterschied zwischen der Vokalartikulation und der Mouillierungsartikulation (und zwischen den entsprechenden akustischen Eindrücken) so gross, dass die Mouillierung nie ausbleibt. Wir finden deshalb in dem Material nie eine solche Verbindung wie z.B. $m \dot{a}$ sondern nur $\dot{m} \dot{a}$. Ausserdem ist vielleicht der Unterschied zwischen den vorderen und den hinteren Vokalvarianten geringer bei $a, o, u$ als derjenige zwischen $i$ und $i$ bzw. $e$ und $e$, sodass ein Oppositionsverhältnis zwischen z.B. $m i$ und $m i$ leichter aufrechtzuerhalten ist als es zwischen ma und $m \dot{a}$ wäre. In dem letzteren Falle wird also $\dot{m} \boldsymbol{a}$ gefordert.

Dass die bisweilen vor $i$ und $e$ ohne Mouillierungszeichen 
auftretenden Konsonanten wirklich zu einer anderen Reihe von Lauten als die vor den hinteren Vokalvarianten stehenden unmouillierten Konsonanten gehören, ist daraus ersichtlich, dass diejenigen Konsonanten, die ausser der Mouillierung auch andere qualitative Unterschiede zwischen der unmouillierten und der mouillierten Form aufzeigen, vor $i$ und $e$ immer in dieser Hinsicht diejenige Qualität haben, die bei dem mouillierten Konsonanten zu Hause ist. So hat der unmouillierte $l$-Laut die Gestalt $t$ aber der mouillierte die Gestalt $l$. Vor $i$ und $e$ kann zwar ausser $l$ auch $l$ stehen, aber nicht 7 . Das Wort für Adler heisst nach WB im Dialekt O limbiè und ' $\mathrm{T}_{1}$ limbe, eine Form *timbe dagegen, mit demselben l-Laut wie in $t \bar{\imath}$ 'Knochen', kommt nicht vor. Diejenigen Konsonanten, die intervokalisch in der unmouillierten Form als $\beta$ und $\delta$ auftreten, haben die mouillierten Entsprechungen $b^{\prime}$ und $d$. Vor $i$ oder $e$ kann ohne Mouillierungszeichen $b$ auftreten, aber nie $\beta$. Für $O s a \bar{\beta} \beta p$ "Mütze» wird der Akk.Pl. $s_{x} \underset{\beta}{\bar{\imath}}$ gegeben (dass der Verschlusslaut hier als stimmlos oder genauer genommen als halbstimmhaft angegeben wird, ist natürlich in diesem Zusammenhang unwesentlich). Es könnte auch sabí (oder

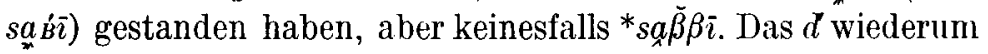
kommt überhaupt nicht ohne das Mouillierungszeichen vor.

Etwas anders verhält es sich mit meiner Schreibung jïde in FO (S. 99) für Lehtisalos jīঠè (MSFOu 90, S. 126), die von Joki (S. 6) nicht ohne Recht als irreführend getadelt wird. Die fragliche Form steht bei Lehtisalo in einer Wortverbindung

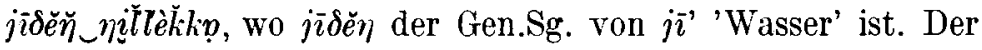
Stamm dieses Wortes ist $j \bar{\imath} \delta$ - (in meiner Schreibung $j \bar{\imath} d-$ ), und der Gen.Sg. ist in der freistehenden, paradigmatischen Form $j \bar{i} \delta^{\prime}$ ( $\left.j \bar{i} d^{\prime}\right)$, wo der Kehlkopfverschluss die Genitivendung ist, die hier in zusammenhängender Rede regelrecht mit dem anlautenden $\eta$ des folgenden Wortes assimiliert wird. Der zwischen $\delta$ und $\eta$ auftretende ganz kurze $e$-Laut ist ein anorganischer, die Aussprache erleichternder Einschubvokal, den ich eigentlich in meiner vereinfachten Transkription hätte fortlassen sollen. Die richtige Schreibung mit dem von mir verwendeten System wäre also in diesem Falle $j \bar{\imath} d \eta$ gewesen.

Noch ein Umstand ist in diesem Zusammenhang hervor- 
zuheben. Auch wenn ein vor $i$ oder $e$ stehender Konsonant nicht als mouilliert bezeichnet ist, hat er auf den vorhergehenden Vokal dieselbe Wirkung wie ein mouillierter Konsonant, d.h. es steht vor ihm diejenige Vokalqualität, die vor einem mouillierten Konsonanten zu erwarten ist, also nicht $a$ sondern $\underset{m}{a}$, nicht $u$ sondern $u$ usw., wie aus den in der obigen Darstellung gegebenen Beispielen zur Genüge hervorgeht.

Aus den angeführten Gründen scheint es mir angebracht, die vor $i$ und $e$ sozusagen gegen die Erwartung ohne Mouillierungszeichen stehenden Konsonanten als spotentiell mouilliert» zu betrachten. Damit meine ich, dass sie systematisch betrachtet zu der Reihe der mouillierten Konsonanten gehören, wenn auch die Mouillierungsartikulation gelegentlich bei ihnen fehlt oder so schwach ist, dass sie nicht aufgezeichnet wurde, denn im Sprachsystem fungieren sie in jeder Hinsicht als mouillierte Konsonanten.

Hierbei muss jedoch ein Sonderfall verzeichnet werden. Das $\chi$ ist ja ein immer unmouillierter Konsonant, und nach anlautendem $\chi$ steht nie $i$ oder $e$, sonder nur $i$ und $\underline{e}$. Recht gewöhnlich sind trotzdem die Iautfolgen $i \chi i$ und exe. Wenigstens in den meisten tundrajurakischen Dialekten ist es eine allgemeine, wenn auch nicht ganz ausnahmslose Regel, dass ein interrokalisches $\chi$ denselben Vokal nach wie vor sich hat. Wenn dabei vor $\chi$ eine vordere (nach mouilliertem Konsonanten stehende) Variante von $a$, o oder $u$ steht, folgt nach dem $\chi$ die nach einem unmouillierten Konsonanten zu erwartende Variante, also z.B. nóozō 'in Schweiss geraten' mit derselben Vokal'fualität der zweiten Silbe wie in noxi' 'Polarfuchs'. Wenn aber vor dem $\chi$ ein $i$ oder $e$ steht, dann kommt auch nach dem $\chi$ dieselbe Vokalqualität wieder, z.B. in O ni ximts's 'verschlingen' und sè xè 'harter Schnee'. Bisweilen wird die $i$-Artikulation etwas nach hinten gezogen, wie in $O \quad j \bar{\imath} \chi \bar{\imath} t$ ' 'Uferwasser im Frühling auf dem Eis', ja man kann auch die normale velare Variante finden, wie in $\mathrm{T}_{\mathbf{g}}$ nixi' s.v. Nj ńixoku 'Barsch'.

Joki verneint auf S. 24 seiner Besprechung von FO das Vorhandensein der genannten Regel. Es gibt wie gesagt Ausnahmen (z.B. O to $\chi \bar{e}^{-}$' 'Winkel' (< ostj.), tò $\chi \bar{e}$ ' 'Kattun' (aber in anderen Mundarten to $\chi \bar{o}$, tò $\left.\chi \hat{o}^{\prime}\right)$, aber sie sind recht spärlich 
und müssen als Sonderfälle betrachtet werden, und jedenfalls sind die von Joki aus Castréns Wörterverzeichnis gegen die Regel angeführten sechs Beispiele nicht gerade glücklich gewählt, denn fünf von ihnen finden sich in Lehtisalos WB wieder und haben dort denselben Vokal nach wie vor dem $\chi$ (wenn man von dem Unterschied zwischen den hinteren und vorderen Varianten der Vokale absieht): 0 lè $\chi \bar{e}$ 'Zwischenraum der gespreizten Beine', nixi 'Kraft', ńò $\chi \bar{o}$ 'in Schweiss

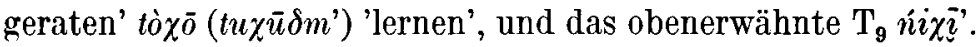

Während wir bei dem $\chi$-Laute sind, möchte ich ganz nebenbei eine interessante Frage berühren, die eher zur Formenlehre gehört. Joki sagt in seiner schon öfters erwähnten Besprechung (\$. 17-18), dass der Akkusativ des Duals nicht mit dem Nominativ identisch sei, wie ich die Sache nach Prokof'ev und T'ereščenko beschrieben habe, sondern dass im Akkusativ der auslautende Kehlkopfverschluss des Nominativs fehle, und er gibt einige Beispiele dafür. Ich habe leider die angeführten Formen weder in Lehtisalos Texten noch in seinen sprachwissenschaftlichen Abhandlungen finden können. Die Dualformen der absoluten Deklination sind überhaupt sehr spärlich vorhanden, denn nach dem Zahlwort für 'zwei' steht gewöhnlich der Singular, und die wirklich vorhandenen Dualformen sind meistens mit einem Possessivsuffix versehen. Beim Durchsehen des WB habe ich überhaupt keine geeignete Beispiele für die Entscheidung der Frage gefunden. Den Nom.Du. habe ich in etwa zehn Fällen angetroffen, und auch ein paar dualische Objekte ohne Possessivsuffix, die auf den Kehlkopfverschluss auslauten, aber es handelt sich dort um imperativische Sätze, wo man den Nominativ als Objektkasus erwarten kann. In Lehtisalos Texten habe ich nur einen einzigen Fall der in Rede stehenden Form gefunden, nämlich auf Seite 7, Zeile 22 der Erzählung: nat'se $\breve{k} k e \chi \bar{e}$, und diese Form hat ja den Kehlkopfverschluss. Der ganze Satz lautet folgendermas-

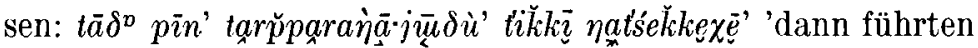
sie die Kinder heraus' (es ist von zwei Kindern die Rede). Unter solchen Umständen ist es sehr zu bedauern, dass Joki die Quelle seiner Belege nicht angegeben hat. Es unterliegt jedenfalls keinem Zweifel, dass der Akk.Du. in der jurakischen 
Schriftsprache mit dem Nom.Du. identisch ist, wie aus den von Prokof'ev und Tereščenko verfassten Grammatiken hervorgeht. Tereščenko gibt in ihrem Buch »Materialy i issledovanija po jazyku nencev) (Moskva-Leningrad 1956) auf Seite 26 die folgenden Beispiele: nanoho' tonaha' '(zwei) Boote kamen' und nanoho' serta" 'sie machten (zwei) Boote'. Es wird bei dem letzteren Satz ausdrücklich angegeben, dass vom $\mathrm{A} \mathrm{k} \mathrm{k} \mathrm{u-}$ s a t i v Dual die Rede ist, und ausserdem wird einige Zeilen später von dem "Kehlkopfverschluss im Nominativ, Genitiv und Akkusativ des Duals" gesprochen.

In seiner Besprechung von FO ist Hajdú (NyK 59, S. 248249) mit mir gegen Joki darüber einig, dass vor den palatalen Vokalen regelmässig mouillierte Konsonanten stehen und dass die Abweichungen, die angetroffen werden, keine prinzipielle Bedeutung haben, aber er beanstandet doch mein Verfahren, in der vereinfachten Transkription das Mouillierungszeichen vor $i$ und $e$ wegzulassen. Er schreibt nämlich: »Jokis Auffassung gründet sich darauf, dass wir auch in Lehtisalos Aufzeichnungen Inkonsequenzen in der Bezeichnung der Palatalisierung finden, aber diese vereinzelten Fälle verändern nicht die allgemeine Erscheinung, dass vor palatalen Vokalen nur palatalisierte Konsonanten auftreten können. Diese Bestrebung charakterisiert die ganze juraksamojedische Sprache, und demzufolge besteht auch das Vokalsystem aus einer vollständigen palatalen und einer vollständigen velaren Serie (jeder einzelne Vokal hat eine palatal und eine velar gebildete Form). Und in Wickmans Transkription ist eben das der Fehler, dass er diese Unterschiede verwischt. Als Beispiel erwähne ich, dass es im Jurakischen einen palatalen $\dot{a}$-Laut und einen velaren $a$-Laut gibt. Es ist angebracht, das $\dot{a}$ und das $a$ als verschiedene Phoneme zu betrachten, und es ist deshalb fehlerhaft, dass Wickman das Wort ńa $\dot{a}$ in der Form ńa wiedergibt. In diesem Falle deutet also das Zeichen der Palatalisierung darauf hin, dass wir es jedoch nicht mit dem Laut $a$ zu tun haben. Gleichzeitig hat der Akk.Pl. dieses Wortes in Wickmans Transkription die Gestalt $n \bar{\imath}$ (richtig $n \hat{n}$ ), und hier bezeichnet - eben umgekehrt - der Vokal die Palatalisierung des Konsonanten. Meines Erachtens ist also die Bezeichnung der Palatalisierung 
bei $\bar{i}$ usw. ebenso wichtig wie bei dem vorangehenden Konsonanten.»

Es ist richtig und ich habe selbst eingesehen, dass man darin eine Inkonsequenz finden kann, dass ich den palatalen Charakter der Lautverbindung in solchen Verbindungen wie $i$ bei dem Vokalzeichen aber bei solchen Verbindungen wie $\dot{n} a$ bei dem Konsonantenzeichen angegeben habe (indem ich $n i$ aber ńa geschrieben habe), und auf diese Frage werde ich gleich zurückkommen, aber sonst kann ich den angeführten Gedankengang Hajdús nicht billigen. Er sagt ja selbst ganz richtig, dass in meiner Schreibung na das Mouillierungszeichen ausser der Palatalisierung des Konsonanten auch diejenige des Vokals angibt, und dass in meiner Schreibung ni das verwendete Vokalzeichen die Palatalisierung des Konsonanten angibt, und folglich ist ja alles vorhanden, was für die eindeutige Bestimmung der in jedem einzelnen Fall vorliegenden Wörter nötig ist. Es scheint aus Hajdús Auseinandersetzung hervorzugehen, dass er sowohl die zwei Reihen von Vokallauten $(a \sim a$ usw.) als die zwei Reihen von Konsonantlauten $(n \sim i$ usw.) als verschiedene Reihen von $\mathrm{Ph}$ o n e m e $\mathrm{n}$ betrachtet. In dem Falle aber sollte man ja erwarten, dass es solche Typen von Gegensätzen mit unterschiedlicher Bedeutung der Glieder wie $n a \sim n \dot{a}$ (und $\dot{n} a \sim n \dot{a}, n i \sim n i, n i \sim n i$ ) gäbe, was er selbst mit vollem Recht leugnet.

Es scheint mir also völlig klar, dass man in einer vereinfachten (und phonematischen) Transkription nicht den palatalisierten oder unpalatalisierten Charakter sowohl der Konsonanten als der Vokale angeben soll. In einer solchen Lautverbindung wie $\dot{n} \dot{a}$ ist entweder $\dot{n}$ eine kombinatorische $\mathrm{Va}$ riante von $n$ oder $\dot{a}$ eine kombinatorische Variante von $a$. Man kann also a priori zwischen zwei verschiedenen Bezeichnungssystemen wählen. Man kann ńa schreiben, so wie ich in FO getan habe, wobei die vordere Aussprache des a-Lautes sich automatisch aus der mouillierten Beschaffenheit des Konsonanten ergibt. Diese Bezeichnungsweise wurde in der früheren jurakischen Schriftsprache verwendet, die das lateinische Alphabet benutzte. Oder aber kann man z.B. na oder 
nä schreiben, wobei die Mouillierung des Konsonanten automatisch daraus folgt, dass der Vokal als palatal bezeichnet ist. Die letztere Methode wird in der heutigen jurakischen Schriftsprache befolgt, die das russische Alphabet benutzt und die als Beispiel gewählte Verbindung ня schreibt, und auch ron Collinder, der in seinen Werken "Fenno-Ugric vocabulary" und "Survey of the Uralic languages» die Bezeichnungsweise $n \mathscr{E}$ verwendet.

Wie schon oben angedeutet wurde, habe ich indessen in FO gewissermassen die beiden soeben beschriebenen Systeme zusammengemischt, als ich die mouillierten Konsonanten als solche vor $a, o, u$ bezeichnete aber nicht vor $i$ und $e$. Ich operiere in FO (nach Prokof'ev) mit demselben Vokalsystem, das oben (S. 104) in dem Zitat von Steinitz dargestellt wurde. Da ich vorziehe, in der systematischen Darstellung die engen, hohen Vokale oben zu setzen und das offene a unten zu placieren, bekommt dieses Vokalsystem das folgende Aussehen:

$\begin{array}{lllll}u & & \stackrel{i}{i} & & i \\ & o & e & e\end{array}$

Es besteht aber kein genügender Grund dafür, unter den illabialen engen und halboffenen Vokalen je ein velares und ein palatales Phonem anzusetzen (d.h. $i$ und $i$ bzw. $\underline{e}$ und $e$ ), bei $a$, $o$ und $u$ dagegen nicht. Wenn $\dot{a}$ eine kombinatorische Variante von $a$ ist usw., dann ist auch $i$ eine kombinatorische Variante von $i$ und $e$ von $e$. Um uns an die gewohnten Buchstabentypen zu halten, ist es in dem Falle angebracht, die Buchstaben $i$ und $e$ zu verwenden und sie in der Stellung nach einem unmouillierten Konsonanten die phonetischen Aktualisierungen $i$ und $e$ bezeichnen zu lassen.

Dem genannten Vorwurf der Inkonsequenz ist das von Collinder verwendete System nicht ausgesetzt, denn er operiert mit einer vollständigen velaren und einer vollständigen palatalen Vokalreihe. Mit den von ihm gebrauchten Zeichen besteht die velare Reihe aus $a, o, \tilde{o}, u, y$ und die palatale aus $x, \infty$, 
$e, \ddot{u}, i$, und in der jurakischen Schriftsprache sind die entsprechenden Zeichen a, о, э, у, ы und я, ё, е, ю, и. Wir würden dann das folgende Vokalsystem bekommen:

$\begin{array}{lllllll}u & & i & & \dot{u} & & i \\ o & & e & & \dot{o} & & e\end{array}$

Die Vokale sind hier ebenso wie in dem auf der vorigen Seite angeführten System in drei Öffnungsgrade eingeteilt, aber hier haben alle Öffnungsgrade (nicht nur der enge und der halboffene) die Einteilung in velare und palatale Vokale, und weiter haben unter den engen und halboffenen Vokalen sowohl die labialen als die illabialen den Gegensatz velar-palatal (m.a.W. es gibt in diesem System auch vordere Labialvokale).

Wenn wir dagegen die mouillierten und unmouillierten Konsonanten als zwei Gruppen von unabhängigen Variablen und die vorderen und hinteren Vokale als abhängige Variablen betrachten, d.h. wenn wir die palatalisierte Beschaffenheit der Konsonanten aber nicht der Vokale bezeichnen, dann können wir mit dem folgenden einfachen Vokalsystem operieren:

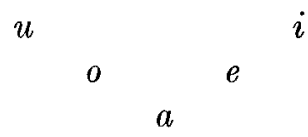

Wie wir schon früher sahen, haben von diesen Vokalen der offene und die engen $(a, u, i)$ phonologisch betrachtet zwei Quantitätsstufen, die halboffenen $(o, e)$ dagegen nicht.

Wir haben jedoch noch nicht die Frage beantwortet, welches von den beiden Systemen vorzuziehen ist. Meines Erachtens kann man für die zuletzt dargestellte Bezeichnungsweise wenigstens die folgenden Argumente heranziehen:

1. Wenn die vordere und die hintere Aussprache der Vokale als verschiedene Phoneme zu betrachten wären, könnte man erwarten, dass sie beide auch im Anlaut vorkämen, wo ja der Einfluss eines vorhergehenden Konsonanten bei isolierter Aussprache ausgeschlossen ist, aber das ist nicht der Fall. Nun 
haben ja bekanntermassen die meisten jurakischen Dialekte normalerweise keinen vokalischen Anlaut, aber in denjenigen Dialekten, die einen solchen normalerweise haben, und auch in denjenigen Wörtern der übrigen Dialekte, die vokalisch anlauten, steht im Anlaut dieselbe Vokalqualität wie nach einem unmouillierten Konsonanten, was darauf deutet, dass das Auftreten eines vorderen Vokals von dem Vorhandensein eines vorangehenden mouillierten Konsonanten abhängig ist. Auch die vorderen $i$ und $e$ kommen im Anlaut nicht vor (sondern nur $i$ und $e$ ), was die Schlussfolgerung bestätigt, dass $i$ und $i$ bzw. $e$ und $e$ nicht als verschiedene Phoneme zu betrachten sind. Die Dialekte, die den velaren sekundären anlautenden Nasal $(\eta$ ) eingebüsst haben, haben den palatalen (ń) bewahrt (wegen Beispiele siehe Hajdús schon erwähnte Untersuchung über die sekundären anlautenden Nasale im Samojedischen).

2. Mouillierte Konsonanten kommen nicht nur vor palatalen Vokalen (und vor anderen mouillierten Konsonanten) vor, sondern auch im Auslaut und vor unmouillierten Konsonanten, und in diesen Stellungen müssen sie unbedingt als selbständige Phoneme betrachtet werden. Auch wenn man für die velaren und die palatalen Vokale verschiedene Zeichen verwendet, muss man also in den erwähnten Stellungen die Mouillierung der Konsonanten in der Schrift angeben, aber das Umgekehrte gilt nicht, sondern wenn man durchgehends die mouillierten Konsonanten als solche bezeichnet, kommt man mit den oben angeführten fünf Vokalzeichen aus. Ausser den häufig im Auslaut vorkommenden mouillierten Konsonanten $s \dot{s}$ und t's (die ja u.a. in der Infinitivendung auftreten, z.B. $t o ̄ s$ ' $k o m m e n '$, mit's 'geben') sind auslautende mouillierte Konsonanten allerdings nicht sehr gewöhnlich. Von vereinzelten Wörtern abgesehen, wie dem unleugbar gewöhnlichen Pronomen man 'ich', kom.men sie in den meisten Fällen in Lehnwörtern vor, z.B $a$ in den russischen Lehnwörtern U, Sjo tārá 'Speisenkiste', tostr 'ungetauft', U sāl 'Kopftuch' und in den ostjakischen Enslehnungen $\mathrm{O}, \mathrm{Lj}, \mathrm{S}, \mathrm{Nj} \beta \bar{a} \bar{l}$ 'Stange' und $\mathrm{O} \mathrm{T}_{1} \mathrm{Sj} \mathrm{K} \mathrm{U}$ Ok MB M Lj ńä́n 'Brot'. Wenn inlautend mouillierte Konsonanten bei der Wortbeugung in Auslaut geraten, werden 
sie meist depalatalisiert. So treten nach Prokof'ev (Jazyki i pis'mennost' narodov severa, I, S. 26) bei der Bildung des Akk.Pl. durch Apokope die folgenden Konsonantenwechsel ein: $l$ geht in $l$ über (z.B. jala 'Tag $\sim$ apl jal), $n$ in $n$ (z.B. tona 'Fuchs' $\sim$ apl ton), $\dot{r}$ in $r$ (z.B. nara 'Spiess' $\sim$ apl nar $)$, $b$ in $w$ (z.B. tiba 'Zahn' apl tiw). Auch im WB kann man Beispiele ähnlicher Art finden, wie O naltè śè 'Schuld' apl natte $\beta$, OP jībe 'Leim' apl $j \grave{i} \beta$. Der oben nach Prokof'ev angeführte Akk.Pl. ton von tóna wird zwar im WB unter dem Stichwort tōn'ne für die Waldjurakischen Dialekte $\mathrm{Nj}$ und $\mathrm{P}$ als t'son̆ angegeben, aber unter dem Stichwort $j \grave{a} \beta t a ̄$ erscheint für den tundrajurakischen Dialekt $\mathrm{Sjo}$ in einem Satze die Form tồn, die allerdings in der Wortverbindung tọn n nōšsi steht, so dass man vielleicht doch nicht davon ganz überzeugt sein kann, dass die Depalatalisierung hier nicht gelegentlich als Sandhierscheinung auftritt. Jedenfalls muss man offenbar im Auslaut in gewisser Ausdehnung mit Phonemzusammenfall der mouillierten und unmouillierten Konsonanten rechnen, wenn man diese beiden Reihen als verschiedene Phonemreihen betrachtet.

3. Das jetzt vorzubringende Argument ist etwas speziellerel Art und betrifft mur den $o$-Laut. Wir haben schon festgestellt, dass dieser Vokal die Variante o nicht nur nach mouillierten Konsonanten hat, sondern auch nach unmouillierten Konsonanten in dem Falle, wenn der folgende Konsonant mouilliert ist, und wir haben also dieselbe Vokalqualität in togs 'kommen' wie in tọñne 'Fuchs' und togr 'Ruf'. Die Qualität $o$ dagegen kommt nur in unmouillierter Umgebung vor, z.B. tōs 'Zobel'. Wenn man nun in einer vereinfachten Transkription den nach mouillierten Konsonanten auftretenden $o$-Laut sagen wir mit $\dot{o}$ wiedergibt (und entsprechend $\dot{a}$ und $\dot{u}$ schreibt) und die Mouillierung der Konsonanten unbezeichnet lässt, dann muss man genau dieselbe Vokalqualität nach unmouillierten Konsonanten mit einem anderen Zeichen wiedergeben (also $o$ ebenso wie $a$ und $u$ ), denn sonst kann nicht mit Sicherheit immer zwischen verschiedenen Wörtern unterschieden werden. Wenn man dagegen die mouillierten und unmouillierten Konsonanten verschiedenartig bezeichnet und die genaue Aussprache der 
Vokale aus der Beschaffenheit der Konsonanten folgen lässt, dann besteht keine Gefahr einer solchen Verwechslung. Wir haben soeben das Wort tōs 'kommen' angeführt. Wenn es nun auch ein Wort tós gibt, wie soll man dann unter den beiden Wörtern unterscheiden können, wenn die Mouillierung des anlautenden Konsonanten nicht in der Schrift wiedergegeben wird? Der angedeutete Ausweg, denselben Laut $g$ in den beiden Wörtern mit verschiedenen Buchstaben wiederzugeben (also etwa $t o s$ und $t \dot{s} s$ ), ist wohl kaum befriedigend. Dieses Dilemma wird beseitigt, wenn man statt dessen toś und toś schreibt.

Ich möchte also die letztere Methode befürworten, und aus schon angeführten Gründen scheint es mir angebracht, auch bei den $i$ - und $e$-Lauten in derselben Weise zu verfahren, so dass der Buchstabe $i$ nach unmouillierten Konsonanten als $i$ und nach mouillierten Konsonanten als $i$ zu lesen ist, der Buchstabe $e$ nach unmouillierten Konsonanten als $e$ und nach mouillierten als $e$. Wenn man dieses System von fünf Vokalphonemen $(a, o, u, e, i)$ verwendet aber z.B. in dem phonetischen Teil einer Grammatik nähere Auskünfte über die tatsächliche Aussprache geben will, muss man natürlich kombinatorische Regeln geben, die in tabellarischer Form folgendermassen ausgedrückt werden kömnen, wobei wir eine Zusammenfassung der obigen Untersuchung über den Vokalismus der ersten Silbe im O-Dialekt bekommen, von gewissen oben behandelten Sonderfällen und Schwankungen abgesehen. Die als Kolumnentitel gebrauchten Symbole geben die jeweilige Stellung des fraglichen Vokals an mit Bezug auf die umgebenden Konsonanten, wobei das Zeichen für einen unmouillierten Konsonanten auch das Fehlen eines Konsonanten bezeichnen kann (d.h. vokalischen Anlaut bzw. Auslaut). In der Tabelle bezeichnet $x$ einen unmouillierten Konsonanten, $\dot{x}$ einen mouillierten Konsonanten und a den in Rede stehenden Vokal. Nur die phonematische Quantität wird angegeben, so dass bei $o$ und $e$ keine Quantitätsunterschiede in Frage kommen. 


\begin{tabular}{|c|c|c|c|c|}
\hline & $x 3 x$ & $x_{3} \dot{x}$ & $\dot{x} 3 x$ & $\dot{x} s \dot{x}$ \\
\hline $\mathbf{a}$ & $a$ & $\underset{n}{a}$ & $\dot{a}$ & $\ddot{a}$ \\
\hline$\overline{\mathbf{a}}$ & $\bar{a}$ & $\vec{a}$ & $\overline{\dot{a}}$ & $\overline{\dot{i}}$ \\
\hline 0 & $o$ & $g$ & $g$ & $o$ \\
\hline $\mathbf{u}$ & $u$ & $u$ & $u$ & $\ddot{u}$ \\
\hline$\overline{\mathbf{u}}$ & $\bar{u}$ & $\bar{u}$ & $\bar{u} \bar{u}$ & $\bar{u}$ \\
\hline $\mathbf{e}$ & $e$ & $\rho$ & $e$ & $e$ \\
\hline i & $i$ & $i$ & $i$ & $i$ \\
\hline i & $\vec{\imath}$ & $\bar{\imath}$ & $\bar{\imath}$ & $i$ \\
\hline
\end{tabular}

Der Diphthong $\breve{a} \dot{e}$, der nur nach unmouillierten Konsonanten

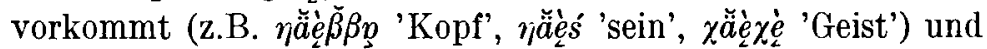

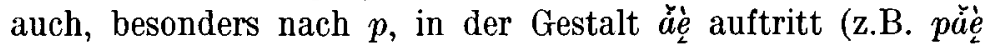
'Stein', päè $\chi \dot{e}$ 'getrockneter Fisch'), und dem in verschiedenen Dialekten $\bar{e}$, ie oder ähnliche hintervokalische Laute oder Lautverbindungen entsprechen, kann phonematisch als ae geschrieben werden.

In Anbetracht des (wenigstens in dem aufgezeichneten Material) recht häufigen Ausbleibens der Mouillierung vor $i$ und $e$ kann die folgende zusätzliche Ausspracheregel gegeben werden: Wenn ein vor $i$ oder $e$ stehender Konsonant mit dem Mouillierungszeichen versehen ist, kann in gewissen Fällen (die oben, S. 114, dargelegt sind) die Mouillierung ausbleiben oder wenigstens sehr schwach sein. Dies hat für denjenigen, der an eine phonetische Bezeichnungsweise gewohnt ist, die vielleicht beim ersten Anblick erstaunliche Folge, dass, sagen wir, ein Graphem $\not i$ phonetisch als $p i$ realisiert werden kann, während ein Graphem $p i$ als $p i$ zu lesen ist. Vor einem mouillierten Konsonanten kann ausserdem z.B. $\dot{p} u$ - als $p \ddot{u}$ - ausgesprochen werden.

In Anbetracht der Sonderstellung des Lautes $\chi$ mit Bezug auf den umgebenden Vokalismus kann ausserdem die folgende Regel gegeben werden, die allerdings nicht den Vokalismus der ersten Silbe betrifft und auch nicht voll und ganz allgemein gültig ist: Wenn zwischen zwei $i$-Lauten oder zwei $e$-Lauten $\chi$ steht, hat der zweite Vokal dieselbe Qualität wie der erste.

Es sei noch mit Bezug auf die ganze hier mitgeteilte Unter- 
suchung die Bemerkung hinzugefügt, dass Lehtisalo in seiner im Jahre 1927 erschienenen Dissertation "Über den vokalismus der ersten silbe im juraksamojedischen" (MSFOu 56) in gewissen Punkten eine von der hier behandelten, abweichende Bezeichnungsweise verwendet, auf die hier nicht eingegangen wird.

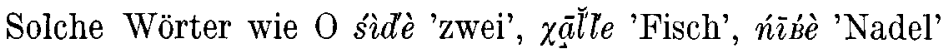
wurden in der früheren jurakischen Schriftsprache (mit lateinischen Buchstaben) als sida, hala, niba geschrieben, und in der heutigen Schriftsprache werden sie (mit russischen Buchstaben) als сидя, халя, нибя geschrieben. Sie werden also als auf einen mouillierten Konsonanten $+a$ auslautend betrachtet.

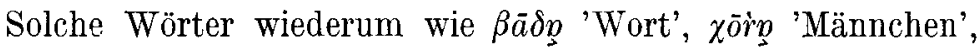
$s a \beta \beta p$ 'gut' wurden früher uada, hora, sawa und werden jetzt вада, хора, сава geschrieben. Beide diese Worttypen werden also als $a$-Stämme aufgefasst, und Prokof'ev (Jazyki i pis'mennost' narodov severa, I, S. 12, Samoučitel' neneckogo jazyka, S. 9) gibt eine Ausspracheregel, nach der ein auslautendes $a$ in zwei- und mehrsilbigen Nominalstämmen eine reduzierte Aussprache bekommt und dabei nach einem unmouillierten Konsonanten ein $ə$ - oder e-ähnlicher Laut aber nach einem mouillierten Konsonanten $e$ wird, während der $a$-Laut in Beugungsformen zum Vorschein kommt. Man vergleiche z.B. einerseits $O \beta \bar{a} \delta p$ 'Wort' $\sim$ Lok.Sg. $\beta \bar{a} \delta \bar{a} \bar{n} n \underline{z}$ und

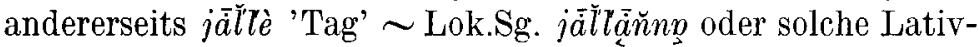

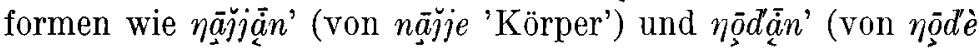
'Beere'). Doch steht der $e$-Laut auch in gewissen Beugungsformen, wie z.B. Akkusativ Singular. Ich behaupte nicht, dass dies Verfahren das beste oder einzig richtige ist, die beiden Worttypen unter der Bennennung a-Stämme zusammenzuführen, aber man kann ihm kaum eine gewisse Berechtigung absprechen, und jedenfalls liegt ein Missverständnis vor, wenn Joki mir in seiner Besprechung (S. 23) vorwirft, zwischen uralischen $a$ - und $\ddot{a}$-Stämmen nicht unterscheiden zu können. Natürlich weiss ich, dass z.B. O ńísè 'Nadel' ein uralischer $\ddot{a}$-Stamm ist (finnisch äimä), aber ich betrachte in meiner 
Abhandlung die Wörter von diesem Typus als jurakische $a$-Stämme, d.h. ich betrachte den mouillierten oder unmouillierten Charakter des vorhergehenden Konsonanten als für die Aussprache des stammauslautenden Vokals ausschlaggebend, wobei also die Aussprachevarianten des Vokals automatisch eintretende Folgeerscheinungen sind. Joki sagt auf Seite 23 seiner Besprechung: "Meiner Auffassung nach hängt der Vokalismus der Endsilbe der Akk.Pl.-Formen hauptsächlich natürlich von dem ursprünglichen (ursamoj.) Vokal des Singularstammes ab (und bei weitem nicht rom Konsonantismus des Wortes, wie es Wickman annimmt).» Die sprachgeschichtliche Beobachtung als solche kann ich ohne weiteres gutheissen, aber bei der Beurteilung meiner Darstellung hat Joki hier die historische und die deskriptive Betrachtungsweise vermischt. Auf S. 85 der Abhandlung gebe ich ja nur für das heutige Jurakische geltende deskriptive Regeln, wie auch Hajdú in seiner Besprechung (S. 250) hervorhebt. Auf S. 88 gebe ich die historische Erklärung dieser Regeln. Dort wird ganz klar zwischen den alten $a$ - und $\ddot{a}$-Stämmen unterschieden, und meine dort ausgesprochene Auffassung stimmt im Wesentlichen mit der von Joki vorgeführten überein, so dass seine auf S. $21-23$ der Besprechung gegebene "vorläufige Mitteilung" mit meiner Darstellung verglichen eigentlich nichts Neues darbietet. Der einzige Punkt dieser "vorläufigen Mitteilung", der nennenswert von meiner Darstellung abweicht, betrifft die ursprünglichen $a$-Stämmen mit langem illabialem Vokal in der ersten Silbe. Nach meinem Schema auf S. 88 sollen diese Wörter im Akk.Pl. Apokope haben, während sie nach Jokis Schema auf S. 21 auf $-i$ auslauten sollen. Sowohl für die ursprünglichen $a$-Stämme mit langem Labialvokal der ersten Silbe wie für die ursprünglichen $\ddot{a}$-Stämme mit langem Vokal der ersten Silbe akzeptiert Joki meine Schlussfolgerung, dass hier die Entwicklung (nach meinem Schema) "*-ai/-äi>ø" stattgefunden hat. Auch betreffs der einsilbigen Stämme, der zweisilbigen mit kurzem Vokal in der ersten Silbe und der dreisilbigen stimmen unsere Ansichten in erfreulicher Weise überein, und bei näherer $\mathrm{Be}$ trachtung wird die ganze von Joki gegebene worläufige Mitteilung" nur eine in gewissen Punkten modifizierte Wieder- 
holung der Resultate meiner in $\mathrm{F}()$ ausgeführten Untersuchung der Frage.

Wenn man, von dem tatsächlichen Unterschied in der Aussprache der zı den beiden Typen gehörigen Wörtern ausgehend, von jurakischen $a$ - und $e$-Stämmen spricht, was sich natürlich rechtfertigen lässt, muss man jedoch bei der historischen und vergleichenden Betrachtung im Gedächtnis behalten, dass die Verteilung der Wörter auf die jurakischen $a$ - und $e$-Stämme nicht durchgehends der Verteilung auf uralische $a$ - und ä-Stämme entspricht. Solche Wörter wie $O \chi \bar{a} \bar{l} l e$ 'Fisch' und niëjje 'Schwiegertochter' müssen, vom ursprünglichen Stammtỵpus unabhängig, im Jurakischen e-Stämme sein, da ein hinterer $a$-Laut hier nicht nach $j$ oder einem mouillierten Konsonanten stehen könnte, und umgekehrt kön-

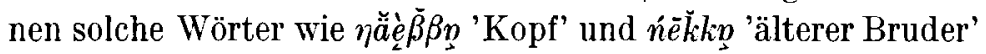
nur jurakische $a$-Stämme sein. Es ist jedoch wahr, dass in den meisten Fällen, wo die Etymologie des Wortes klar ist und der ursprüngliche Stammtypus sich feststellen lässt, die jurakischen $a$-Stämme auf uralische $a$-Stämme und die jurakischen e-Stämme auf uralische $\ddot{a}$-Stämme zurückzuführen sind, und in den meisten Fällen ist wohl historisch betrachtet der Grund der Mouillierung des Stammkonsonanten in den jurakischen e-Stämmen eben der ursprüngliche Vordervokal im Stammauslaut, aber deskriptiv betrachtet ist im heutigen Jurakischen nach meiner Auffassung wie gesagt die mouillierte oder unmouillierte Beschaffenheit des Konsonanten der primäre Faktor und die Vokalqualität davon abhängig. Warum einige Wörter von der Gruppe der $a$-Stämme in die der e-Stämme (oder umgekehrt) übergegangen sind, ist wohl noch nicht immer ganz klar, und diese Frage sollte näher untersucht werden. Es ist interessant zu beobachten, dass in gewissen Fällen die Palatalisierung des Wortes sich über die Endsilbe hinaus auch zur ersten Silbe ausgebreitet haben kann, was augenscheinlich in dem Worte $O_{1}$ tuinnìe 'Feuer anmachen' geschehen ist. Dieses Wort ist wohl eine Ableitung von $t \bar{u}$ 'Feuer' und würde vielleicht im Finnischen mit dem entsprechenden Ableitungssuffix *tulentaa heissen. Lönnrot hat das intransitive Verb tulentua 'feurig, glühend, verbrennt, brennend, heiss werden', und es gibt ja 
sulche Piarre wie pahentaa 'verschlechtern, schlechter machen' pahentua 'sich verschlechtern, schlechter werden'. Es kann bemerkt werden, dass das jurakische Verb auch transitiv, mit Objekt gebraucht wird, was z.B. aus dem im WB gegebenen Beispiel 'I' $t \bar{u} r$ t'uñńé $\delta$ ! 'mache Feuer!' hervorgeht, also etwa in der Bedeutung 'brennend machen', wie man ja dies auch von einem finnischen Verb *tulentaa erwarten würde. Die Zusammensetzung tüñni 'Flinte' hat dagegen das unmouillierte $t$ beibehalten. Die Zusammensetzung der etymologisch entsprechenden Bestandteile würde im Finnischen tulinuoli 'Feuerpfeil' ergeben, und es sei der Kuriosität halber erwähnt, dass dieses Wort tatsächlich von Lönnrot in sein Wörterbuch aufgenommen wurde (mit der schwedischen Übersetzung 'elddrake, eldkula').

Bo Wickman 